\title{
Factors Associated with the Effectiveness of a Telephone-Based Nursing Strategy for Enhancing Medication Adherence in Schizophrenia
}

\author{
José Manuel Montes ${ }^{1}$, Jorge Maurino ${ }^{2}$, , Teresa Diez ${ }^{2}$ and Jerónimo Saiz-Ruiz ${ }^{3}$ \\ ${ }^{I}$ Department of Psychiatry, Hospital del Sureste, Arganda del Rey, Madrid, Spain; ${ }^{2}$ AstraZeneca Medical Department, \\ Madrid, Spain; ${ }^{3}$ Department of Psychiatry, Hospital Universitario Ramón y Cajal, Madrid, Spain
}

\begin{abstract}
A post hoc analysis was made to identify factors associated with success following a 4-month telephone-based strategy for enhancing adherence to antipsychotic treatment in schizophrenia. A total of 928 stable outpatients were randomized to receive a monthly telephone call provided by a nurse or routine clinical care. Logistic regression with a backward stepwise procedure was used. A higher percentage of patients in the intervention group $(25.7 \%, \mathrm{n}=109)$ improved adherence at the end of the study compared with the control group $(16.8 \%, \mathrm{n}=74)(\mathrm{p}=0.0013)$. The intervention was significantly associated with adherence improvement in those patients with a previous negative attitude towards medication $(\mathrm{OR}=4.7,95 \% \mathrm{CI}=2.4-9.0, \mathrm{p}<0.0001)$. A slight concordance was obtained between adherence improvement and improvement in patient perception of treatment ( $\mathrm{kappa}=0.21 ; 95 \% \mathrm{CI}=0.15-0.27)$. The identification of factors related to the effectiveness of a specific intervention would offer clinicians the opportunity to more adequately select patients who are eligible for such intervention.
\end{abstract}

Keywords: Schizophrenia, medication adherence, mental health nursing, telephone, antipsychotic drugs.

\section{FINDINGS}

Antipsychotic medication is the primary treatment modality for acute episodes as well as for relapse prevention in schizophrenia [1]. However, the full benefits of medication as demonstrated by clinical research trials have failed to become implemented in routine clinical care. Poor adherence to medication is thought to be a significant obstacle in achieving this goal $[2,3]$. Up to $75 \%$ of patients with schizophrenia discontinued treatment within two years of hospital discharge [4]. Non-adherence to treatment is associated with poorer clinical and functional outcomes, increased use of emergency psychiatric services, and an increased number of hospitalizations [5].

Many intervention strategies have been used to improve medication adherence in patients with schizophrenia [6]. In a recently published randomized, controlled, four-month study, an intervention based on a telephone call provided by a mental health nurse once monthly proved effective in enhancing oral antipsychotic adherence among patients with schizophrenia [7].

Non-adherence may be determined by many factors that could contribute to maintain patients in this situation even after intervention for improving adherence [2]. Identifying risk factors associated with failure after a specific intervention for enhancing adherence in patients with schizophrenia would allow improvement of the intervention. On the other hand, the identification of factors related to the success of a specific intervention would offer clinicians the opportunity

*Address correspondence to this author at the Serrano Galvache, 56 Parque Norte, Edificio Roble (28033) Madrid, Spain; Tel: +34 913019738;

Fax: +34 913019606; E-mail: jorgealejandro.maurino@astrazeneca.com to more adequately select patients who are eligible for such intervention.

We performed a post hoc analysis using data from the aforementioned study [7]. The aim was to identify factors associated with success following a 4-month telephonebased nursing strategy for improving adherence to maintenance antipsychotic treatment in patients with schizophrenia.

The study was conducted in 198 community mental health centers throughout Spain. Ethical approval was obtained from the Fundació Catalana d'Hospitals (Barcelona, Spain). Data were collected from October 2006 to November 2007. After complete description of the study to the participants, written informed consent was obtained. The sample population for analysis comprised a total of 865 clinically stable outpatients having a DSM-IV-TR diagnosis of schizophrenia, being at least 18 years old, and taking a single oral antipsychotic drug. Their mean age was 40.08 years (SD 11.6), and $65.3 \%$ were males. The mean baseline Clinical Global Impression - Severity score was 3.2 (SD 1.2). The patients randomized to the intervention group $(n=424)$ received a standardized telephone call made by a nurse at weeks 4,8 , and 12 . Patients assigned to the control group $(n=441)$ were scheduled for the final visit four months after inclusion, without telephone calls, and emulating the usual period of clinical care among stable outpatients with schizophrenia in Spain. Each telephone call consisted of a brief semi-structured assessment of medication adherence (a series of questions inquired about knowledge of the medication regimen and episodes of missed doses or incomplete adherence). In addition, the interview included the Drug Attitude Inventory in its 10-item version (DAI-10) [8]. Nurses received specific training for the study from investigators before the first patient contact. 


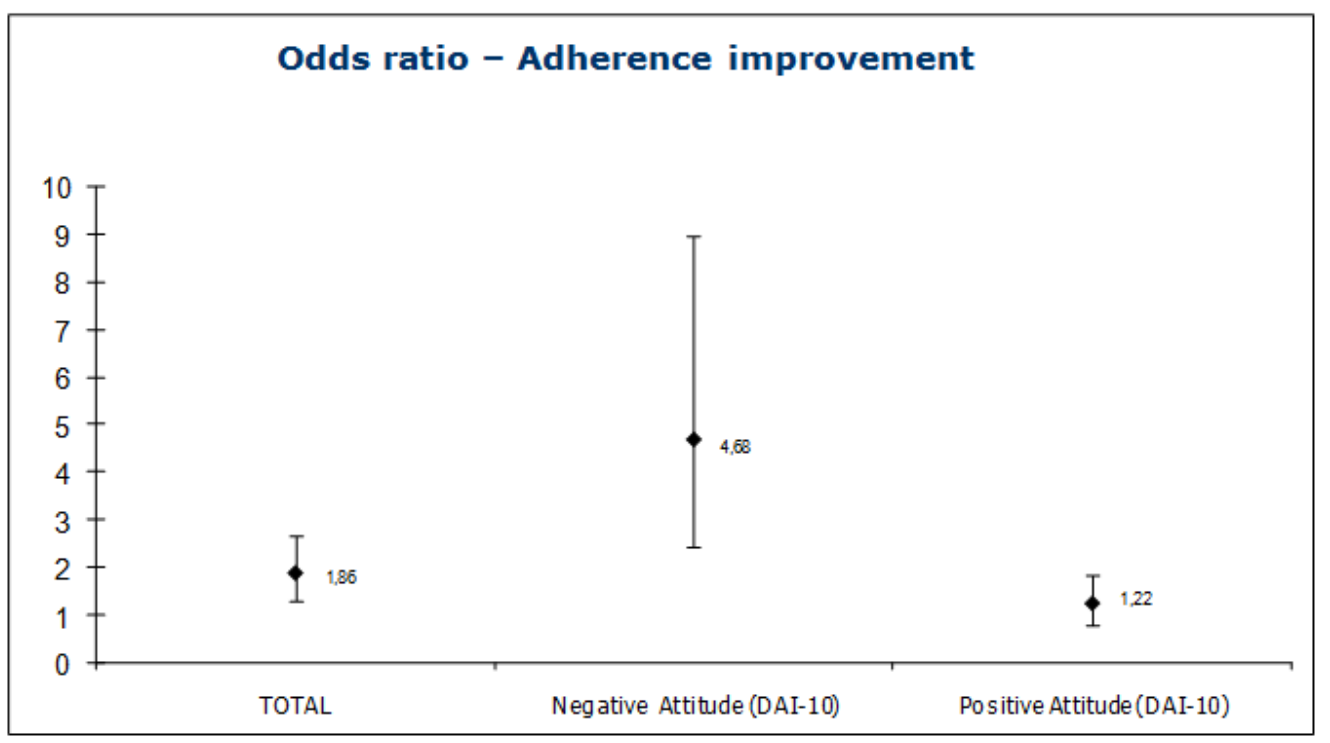

Fig. (1). Logistic regression for adherence improvente according to DAI-10

Adherence to antipsychotic treatment was evaluated by the treating psychiatrist and the nurse according to the Register of Adherence to Treatment scale (RAT): high (adherence with $80 \%$ of doses), moderate (adherence with $60-79 \%$ of doses), low (adherence with $20-59 \%$ of doses), and nil (adherence with $<20 \%$ of doses) [7]. For the analysis, an improvement in adherence to treatment was considered when a patient was at least in an immediately higher level of adherence according to the RAT at the end of the study compared to baseline (e.g., moderate adherence at the end of study vs. low adherence at baseline).

A significantly higher percentage of patients in the intervention group $(25.7 \%, n=109)$ improved adherence to treatment at the end of the study compared with the control group $(16.8 \%, \mathrm{n}=74)(\mathrm{p}=0.0013)$.

To explore factors which could be related to improvement in adherence after the intervention, socio-demographic and clinical characteristics of the patients who improved their adherence status were compared with those who did not. No significant differences were observed in terms of age, gender, living status, education, marital and occupational status, schizophrenia subtypes, number of hospitalizations, class of antipsychotic drug (typical vs. atypical) or previous psychoeducation received. On the other hand, a significantly shorter duration of illness (11.6 vs. 13.5 years; $\mathrm{p}=0.0196)$, a greater number of previous acute episodes $(2.3$ vs. 1.6; $\mathrm{p}<0.0001)$, and shorter periods of time from the last acute episode $(1.9$ vs. 3.5 years; $p<0.0001)$ or from last hospitalization ( 2.9 vs. 4.9 years; $p<0.0001)$ were observed in those patients who improved their adherence status at the end of the study compared to those patients who did not experience improvement. Taking into account the bivariate analysis described above, a multivariate analysis (logistic regression) was performed to more adequately identify outcome predictors for improvement in adherence. Sociodemographic and clinical variables were introduced in the model as independent variables, with improved adherence as the dependent variable. After backward stepwise elimination of the non-significant variables $(p>0.05)$, the following vari- ables remained in the model: group of study (intervention vs. control, $\mathrm{OR}=1.86,95 \% \mathrm{CI}=1.31-2.65, \mathrm{p}=0.0006)$ and baseline DAI-10 score (negative [score <0] vs. positive attitude towards medication [score $>0$ ], $\mathrm{OR}=5.57,95 \% \mathrm{CI}=3.83$ $8.11, \mathrm{p}<0.0001)$. The odds ratio for improvement in adherence with the intervention was calculated in those patients with baseline negative and positive attitudes towards medication. The telephone-based nursing intervention was significantly associated with improved adherence in those patients with a previous negative perception of treatment $(\mathrm{OR}=4.7$, CI $95 \%=2.4-9.0, \mathrm{p}<0.0001)$, but there was no significant association in those patients with a previous positive perception $(\mathrm{OR}=1.2,95 \% \mathrm{CI}=0.8-1.9, \mathrm{p}=0.3628)$ (Fig. 1). A kappa index was obtained to assess the concordance between the improvement in adherence after the intervention and improvement in patient perceptions of treatment. A slight concordance was observed between these two variables $(\mathrm{k}=0.21$; $95 \% \mathrm{CI}=0.15-0.27)$.

The principal findings from these analyses demonstrate that the intervention was significantly more effective in those patients with a previous negative perception of treatment compared to those with a good perception.

The critical importance of optimal adherence to prescribed antipsychotic treatments has been convincingly demonstrated in patients with schizophrenia [4]. Positive attitude towards medication, as measured by the DAI-10, is associated with significantly higher adherence rates [9].

This study suggests that adherence can be improved with an intervention even in those patients with a risk factor consistently associated to non-adherence, such as a negative attitude towards medication. Furthermore, when a bad perception of antipsychotic treatment is detected in a patient, this telephone intervention could be applied in order to enhance adherence and, subsequently, to improve the outcome.

Improvement in adherence after the intervention in those patients with a baseline negative perception of treatment could be partially explained by improvement in their attitude towards medication. A slight concordance was observed be- 
tween these two variables, suggesting that in some patients the telephone call improved the perception of antipsychotic treatment. Alternatively, in those patients who enhanced adherence without improving their attitude towards medication, the intervention could be acting as a reminder, increasing the number of days patients are taking the medication as prescribed. We can hypothesize that patients increasing adherence will improve their attitude towards medication over the long term, as a consequence of their psychopathological improvement.

A limitation of this analysis was the lack of assessment of some factors consistently related to non-adherence. A measure for insight was lacking in the study and factors related to medication, with the exception of the class of antipsychotic drug, were not systematically assessed. In addition, other environmental factors that are emerging as being predictive of non-adherence, including a poor alliance with the therapist or clinician, or less outpatient contact, were not assessed [10]. Consequently, the benefit of the intervention in non-adherent patients presenting these risk factors may not be established.

In summary, the results of this analysis reveal that a telephone call made by a mental health nurse is a successful intervention for enhancing adherence in stable patients with schizophrenia. This intervention could be especially useful for improving adherence in those patients with a negative attitude towards antipsychotic treatment.

\section{STATEMENT OF INTEREST}

This study was funded by AstraZeneca Spain. JM and TD are employees of AstraZeneca.

\section{REFERENCES}

[1] Vohora D. Atypical antipsychotic drugs: current issues of safety and efficacy in the management of schizophrenia. Curr Opin Investig Drugs 2007; 8: 531-8.

[2] Julius RJ, Novitsky MA, Dubin WR. Medication adherence: a review of the literature and implications for clinical practice. J Psychiatr Pract 2009; 15: 34-44.

[3] Jonsdottir H, Opjordsmoen S, Birkenaes AB, et al. Medication adherence in outpatients with severe mental disorders: relation between self-reports and serum levels. J Clin Psychopharmacol 2010; 30: 169-75.

[4] Velligan DI, Weiden PJ, Sajatovic M, et al. The expert consensus guideline series: Adherence problems in patients with serious and persistent mental illness. J Clin Psychiatry 2009; 70 (suppl 4): 1-46.

[5] Ascher-Svanum H, Faries DE, Zhu B, Ernst FR, Swartz MS, Swanson JW. Medication adherence and long-term functional outcomes in the treatment of schizophrenia in usual care. J Clin Psychiatry 2006; 67: 453-60.

[6] Dolder CR, Lacro JP, Leckband S, Jeste DV. Interventions to improve antipsychotic medication adherence: review of recent literature. J Clin Psychopharmacol 2003; 23: 389-99.

[7] Montes JM, Maurino J, Diez T, Saiz Ruiz J. Telephone-based nursing strategy to improve adherence to antipsychotic treatment in schizophrenia: a controlled trial. Int J Psychiatr Clin Pract 2010, 14: 274-81.

[8] Robles García R, Salazar Alvarado V, Paez Agraz F, Ramírez Barreto F. Assessment of drug attitudes in patients with Schizophrenia: psychometric properties of the DAI Spanish version. Actas Esp Psiquiatr 2004; 32: 138-42.

[9] Liu-Seifert H, Osuntokun OO, Godfrey JL, Feldman PD. Patient perspectives on antipsychotic treatments and their association with clinical outcomes. Patient Prefer Adherence 2010; 4: 369-77.

[10] Lacro JP, Dunn LB, Dolder CR, et al. Prevalence of and risk factors for medication non-adherence in patients with schizophrenia: a comprehensive review of recent literature. J Clin Psychiatry 2002; 63: 892-909.

Received: December 03, 2010

Revised: March 03, 2011

Accepted: March 14, 2011

(C) Montes et al.; Licensee Bentham Open.

This is an open access article licensed under the terms of the Creative Commons Attribution Non-Commercial License (http://creativecommons.org/licenses/by-nc/3.0/) which permits unrestricted, non-commercial use, distribution and reproduction in any medium, provided the work is properly cited. 